\author{
Heiner Frei $\cdot$ Regula Everts $\cdot$ Klaus von Ammon \\ Franz Kaufmann - Daniel Walther \\ Shu-Fang Hsu-Schmitz • Marco Collenberg \\ Katharina Fuhrer $\cdot$ Ralph Hassink • Maja Steinlin \\ André Thurneysen
}

\title{
Homeopathic treatment of children with attention deficit hyperactivity disorder: a randomised, double blind, placebo controlled crossover trial
}

Received: 8 March 2005/ Revised: 23 May 2005 / Accepted: 1 June 2005 / Published online: 27 July 2005

(C) Springer-Verlag 2005

\begin{abstract}
An increasing number of parents turn to homeopathy for treatment of their hyperactive child. Two publications, a randomised, partially blinded trial and a clinical observation study, conclude that homeopathy has positive effects in patients with attention deficit hyperactivity disorder (ADHD). The aim of this study was to obtain scientific evidence of the effectiveness of homeopathy in ADHD. A total of 83 children aged 6-16 years, with ADHD diagnosed using the Diagnostic and Statistical Manual of Mental DisordersIV criteria, were recruited. Prior to the randomised, double blind, placebo controlled crossover study, they were treated with individually prescribed homeopathic medications. 62 patients, who achieved an improvement of $50 \%$ in the Conners' Global Index (CGI), participated in the trial. Thirteen patients did not fulfill this eligibility criterion (CGI). The responders were split into
\end{abstract}

H. Frei

Swiss Association of Homeopathic Physicians SAHP,

Lucerne, Switzerland

R. Everts · F. Kaufmann · D. Walther $\cdot$ K. Fuhrer

M. Steinlin $(\square)$

Division of Paediatric Neurology, University Children's Hospital, Inselspital, 3010 Berne, Switzerland

E-mail: maja.steinlin@insel.ch

Tel.: +41-31-6329424

Fax: + 41-31-6329500

K. von Ammon $\cdot$ A. Thurneysen

Kollegiale Instanz für Komplementärmedizin

(KIKOM)/Homeopathy, Imhoof Pavillon,

Inselspital, 3010 Berne, Switzerland

E-mail: andre.thurneysen@kikom.unibe.ch

Tel.: + 41-31-6329758

Fax: + 41-31-6324263

S.-F. Hsu-Schmitz $\cdot$ M. Collenberg

Department of Mathematical Statistics and Actuarial Science

(IMSV), University of Berne, Berne, Switzerland

R. Hassink

Zentrum für Entwicklungsförderung und pädiatrische

Neurorehabilitation, Bienne, Switzerland two groups and received either verum for 6 weeks followed by placebo for 6 weeks (arm A), or vice-versa (arm B). At the beginning of the trial and after each crossover period, parents reported the CGI and patients underwent neuropsychological testing. The CGI rating was evaluated again at the end of each crossover period and twice in long-term follow-up. At entry to the crossover trial, cognitive performance such as visual global perception, impulsivity and divided attention, had improved significantly under open label treatment $(P<0.0001)$. During the crossover trial, CGI parentratings were significantly lower under verum (average 1.67 points) than under placebo $(P=0.0479)$. Long-term CGI improvement reached 12 points $(63 \%, P<0.0001)$. Conclusion: The trial suggests scientific evidence of the effectiveness of homeopathy in the treatment of attention deficit hyperactivity disorder, particularly in the areas of behavioural and cognitive functions.

Keywords Attention deficit hyperactivity disorder · Homeopathy · Individualised treatment · Randomised double blind trial $\cdot$ Treatment effectiveness

Abbreviations ADHD: attention deficit hyperactivity disorder · CGI: Conners' global index · CPRS: Conners' parent rating scale (long form) - CTRS: Conners teacher rating scale $\cdot$ DSM-IV: diagnostic and statistical manual of mental disorders - K-ABC: Kaufman assessment battery for children QCB: questionnaire of change of behaviour - TAP: test battery for attention performance VLMT: German version of the Rey auditory verbal learning test (RAVLT) - WISC-III: German version of Wechsler intelligence scale for children

\section{Introduction}

The attention deficit hyperactivity disorder (ADHD/ ADD) is a combination of disturbed attention (visual, 
tactile, auditory and proprioceptive) and hyperactivity/ impulsivity or passivity. Diagnostic criteria for the disorder are six or more symptoms of either inattention, hyperactivity/impulsivity, or both [2]. ADHD is one of the most common disorders of childhood, occurring in $3 \%-5 \%$ of children, with male to female ratios ranging from $3: 1$ to $9: 1$ [16]. Common medical treatments are stimulants which fall under the legislation for narcotics. Parents who do not want such medication for their child increasingly seek homeopathic alternatives $[5,10]$. Homeopathy is claimed to influence disease using the effect of highly diluted substances, chosen according to the characteristic individual symptoms of the patient. The aim of the homeopathic physician is to find the specific remedy that corresponds accurately to the individual symptoms of the patient [11]. Since this process depends on precise observations by patients or parents, the initial phase of treatment is often characterised by the use of different medications until an optimal response is reached. The effectiveness of homeopathy, in which the active substance is no longer traceable due to its high dilution, is controversial.

Positive effects of homeopathy on ADHD symptoms have been observed in a randomised, partially blinded, placebo controlled crossover trial with 43 children by Lamont [17] who found significant treatment effects. In an observation study with open label treatment, Frei and Thurneysen [8] observed in 86 of 115 children an improvement of $55 \%$ in the Conners' Global Index (CGI) rated by parents. Of the patients in this study, $22 \%$ needed methylphenidate and 3\% reacted neither to homeopathy nor methylphenidate [8]. A criticism of these trials is that, as they were not completely blinded, there is no scientific evidence that the improvements observed were the result of a treatment effect. Two large meta analyses have, however, concluded that the clinical effects of homeopathic treatments should not just be interpreted as a placebo effect $[15,20]$. The unsatisfactory methodology of many of the studies analysed have, however, been criticised. The suggestion that better trials in specific clinical diseases are necessary to establish scientific evidence for the effectiveness of homeopathy led to the present study.

\section{Objectives}

The primary objective was to investigate whether homeopathy is significantly distinct from placebo in the treatment of ADHD. Neuropsychological tests best suited to detect differences in children's performance during the trial were explored. Secondary objectives were to define the time needed for satisfactory amelioration of symptoms (i.e. to reach eligibility for the crossover trial) and to assess the effectiveness of longterm treatment.

\section{Methods}

\section{Design}

The need for individually prescribed homeopathic medication complicates the planning of a double blind clinical trial, as blinding is only possible after the correct individual medication for each patient has been found. Since this process is particularly difficult in ADHD patients, a straightforward randomised controlled trial was considered impossible. In our previous study [8], we made the observation that in early phase of ADHD treatment with Q-potencies (Liquid homeopathic medications produced by dilution steps of 1:50,000, administered daily [22]), the improvement attained declines within 4 weeks after stopping the medication and reappears if treatment is restarted. The deterioration seen when early treatment is interrupted could therefore be used to test the difference between placebo and verum in a crossover trial and led to the present study design. In the first phase of this study, children received individually prescribed homeopathic treatment (screening phase). Children who achieved a pre-defined level of improvement then participated in a randomised, double blind, placebo controlled crossover trial, to study deterioration under placebo.

The double blind part of the study consisted of two parallel groups of children who received verum $(\mathrm{V})$ for 6 weeks followed by placebo (P) for 6 weeks ( $\operatorname{arm~A:~VP),~}$ or placebo for 6 weeks followed by verum for 6 weeks (arm B: PV). Both arms received another 6 weeks of open label treatment (arm A: VPV, arm B: PVV) and an additional long-term follow-up under treatment. The length of each crossover period was determined using the data from the previous study [8] and a preliminary study in four children which revealed that the carry-over effect was longer than 4 weeks after stopping treatment. Since a carry-over effect may be an obstacle for a crossover trial, relatively long crossover periods of 6 weeks were chosen. From previous experience, this duration was thus considered long enough to diminish this problem. No wash-out time was therefore incorporated between the two crossover periods.

\section{Eligibility}

Eligibility criteria for screening phase: age between 6 and 16 years, both genders, confirmed ADHD according to criteria of the Diagnostic and Statistical Manual of Mental Disorders (DSM)-IV [2] and known neuropsychological correlates (greater difficulty in learning, memory, non-automated language tasks, and traditional frontal executive measures) [6,23], necessity for treatment, absence of any chronic physical, neurological or psychiatric disorders. Necessity for treatment was assumed if parents or teachers imperatively demanded an intervention in order to improve the integration and 
performance of the patient in the family or school. A primary selection of possible candidates was made using the Conners' Parent Rating Scale (CPRS), the Conners' Teacher Rating Scale (CTRS) [7] and the Kinsbourne Attention Questionnaire [14]. Patients complying with the ADHD criteria in these questionnaires underwent neuropsychological and neurological testing at the Division of Paediatric Neurology of the University Children's Hospital Berne to confirm the ADHD diagnosis. Subjects taking methylphenidate were obliged to stop medication before neuropsychological testing. Candidates not conforming to rigorous ADHD criteria in the questionnaires were excluded. Patients were recruited between January 2002 and September 2003 by referral of informed paediatricians and by information of regional ADHD parent organisations. All parents previously knew about their participation in a study involving homeopathy.

\section{Assessment for confirmation of diagnosis}

A 2- to $4 \mathrm{~h}$ neuropsychological examination was performed on patients by a paediatric psychologist and a paediatric neurologist between January 2002 and September 2003. The tests were always arranged in the same order. The organisation of the session and the time of day for testing were identical for all subjects in order to avoid effects of uncontrolled factors on performance. The investigation included the following tests: Checklist DSM-IV criteria [2], Kaufman Assessment Battery for Children (K-ABC) [13] or German version of Wechsler Intelligence Scale for Children (WISC-III) [27], Test battery for Attention Performance (TAP) [30], the Largo motor function test [18] and general physical and neurological examination. Children conforming to all diagnostic ADHD criteria, including a parent-rated CGI [7] of at least 14 points without any treatment, were referred to the homeopathic paediatrician for individual treatment.

\section{Eligibility criteria for crossover trial}

Patients must reach an amelioration of $50 \%$ of the initial CGI value or at least 9 points during the screening phase. Written informed consent was obtained from the parents of each child.

\section{Treatment and assessments}

Screening phase: Patients entered homeopathic treatment within 1 month after confirmation of diagnosis. Every eligible child was seen only once at the beginning of the screening phase by the homeopathic physician to minimise any further psychological support influencing ADHD symptoms. They received an individually prescribed homeopathic treatment daily according to the guidelines described by Hahnemann [11] and
Boenninghausen [3]. Any other ADHD treatment was prohibited throughout the whole study period and had to stop immediately or subsequently. Compliance to this rule was checked by periodical inquiry during treatment monitoring and neuropsychological assessments. Treatment progress was clinically assessed with parents only, at intervals of 4 weeks by the homeopathic paediatrician including regular reporting of the CGI. No other counselling took place during these sessions. Adjustment of the medication was made as necessary. The duration of the screening phase was unlimited.

Crossover trial: Participants eligible for the crossover trial were referred to the University Children's Hospital between September 2002 and February 2004 and assigned at random to treatment sequence arm A or B. Patients, their parents, the investigators and the treating physician were blind to the assigned treatments and the treating physician had no contact with patients and parents during the crossover trial. Children with acute diseases, serious accidents and severe social changes in their life during the crossover trial were not assessed for all endpoints and were considered as dropouts; they were, however, included in the primary endpoint analysis according to the intention-to-treat principle.

Open label treatment follow-up: At the end of the second crossover period, all patients continued on open label verum-treatment for an unlimited period of time.

\section{Endpoints}

The primary endpoint for the crossover trial was the parents' rating of CGI, a 10-item rating scale containing the most important ADHD symptoms (i.e. temper outbursts/excitable, impulsive/overactive/cries often/ inattentive/fidgeting/disturbs other children/easily frustrated/fails to finish things/moods change quickly. Rating: $0=$ never, $1=$ occasionally, $2=$ often, $3=$ very often) [7]. Baseline value for the crossover trial was the CGI assessed after the screening phase. The CGI and the Questionnaire of Change of Behaviour (QCB) as well as VLMT and subtests of WISC-III, K-ABC, Verbal Learning Test (VLMT) [12] and TAP were evaluated at the beginning of the crossover trial, after each crossover period and 6 weeks after the crossover trial. To minimise learning effects, only a few of these tests were identical with the diagnostic evaluation. The Connors' questionnaire was completed again by parents and teachers 14 weeks after the crossover trial and a final CGI was assessed by the homeopathic paediatrician between April 2004 and September 2004 independent of the child's current state.

\section{Ethics}

The study was conducted in accordance with the International Conference on Harmonisation, Guidelines for Good Clinical Practice [19], the Declaration of Helsinki 
with all its amendments [29], and in compliance with relevant regulations for informed consent and protection of subject rights in Switzerland. The study protocol was approved by the ethics committee of the Canton of Berne and Swissmedic. The sponsors had no influence on the design, implementation and publication of the study.

\section{Statistics}

The sample size was calculated for the CGI, the primary endpoint in the crossover trial. A mean reduction of 11.25 points (SD 4.00) in CGI had been observed in a previous homeopathic study [8]. Four weeks after interruption of treatment, the CGI increased by only 5 points (unpublished observation), indicating a possible carry-over effect of verum. If this effect were strong, measurements obtained during the second period should be ignored [9]. A statistical test of carry-over effect is, however, not recommended [24]. To take into account the potential carry-over effect and drop-outs, the sample size was therefore estimated using the conservative approach of a $t$-test for two parallel groups in the first period. A reduction of 5 points from pre-treatment value due to verum was considered clinically relevant and a zero point reduction was assumed under placebo. Taking into account the possibly greater heterogeneity in this trial due to a different recruitment practice when compared with the previous study, the SD was inflated to 5 for verum and the same value was assumed for placebo. To have a 5\% significance level and a 95\% statistical power, 27 patients were required in each treatment arm. Hence, a total of approximate 50-60 patients was planned. No interim analyses were considered.

Patient stratification by age and CGI before treatment start was used in the crossover trial: stratum 1: age 6-11 years, baseline CGI 14-21; stratum 2: age 6-11 years, baseline CGI 22-30; stratum 3: age 12-16 years, baseline CGI 14-21; stratum 4: age 12-16 years, baseline CGI 22-30. For each stratum, a randomisation list for 50 patients was computer-generated with a block size of 4 by the IMSV at the University of Berne. The random treatment assignments were sealed in consecutively numbered envelopes and given to the manufacturer Spagyros, who produced the homeopathic medications [11] and their placebos. Spagyros was informed in writing by the treating physician when a child was eligible for the double blind crossover study. The appropriate medication for that child was then prepared according to the randomisation scheme. The medication was sent by mail to the participating family at the beginning of each crossover period. Placebos $(20 \%$ alcohol) were indistinguishable from verum in packaging, labelling, colour and taste. There was no contact between Spagyros, the investigators, the participants and the treating physician during the crossover trial, except when a child dropped out due to unexpected problems.
Fig. 1 Participant flow chart. Left side: recruitment and selection of participants for screening phase. Participant flow during screening phase and crossover trial. Right side: dropouts at all levels

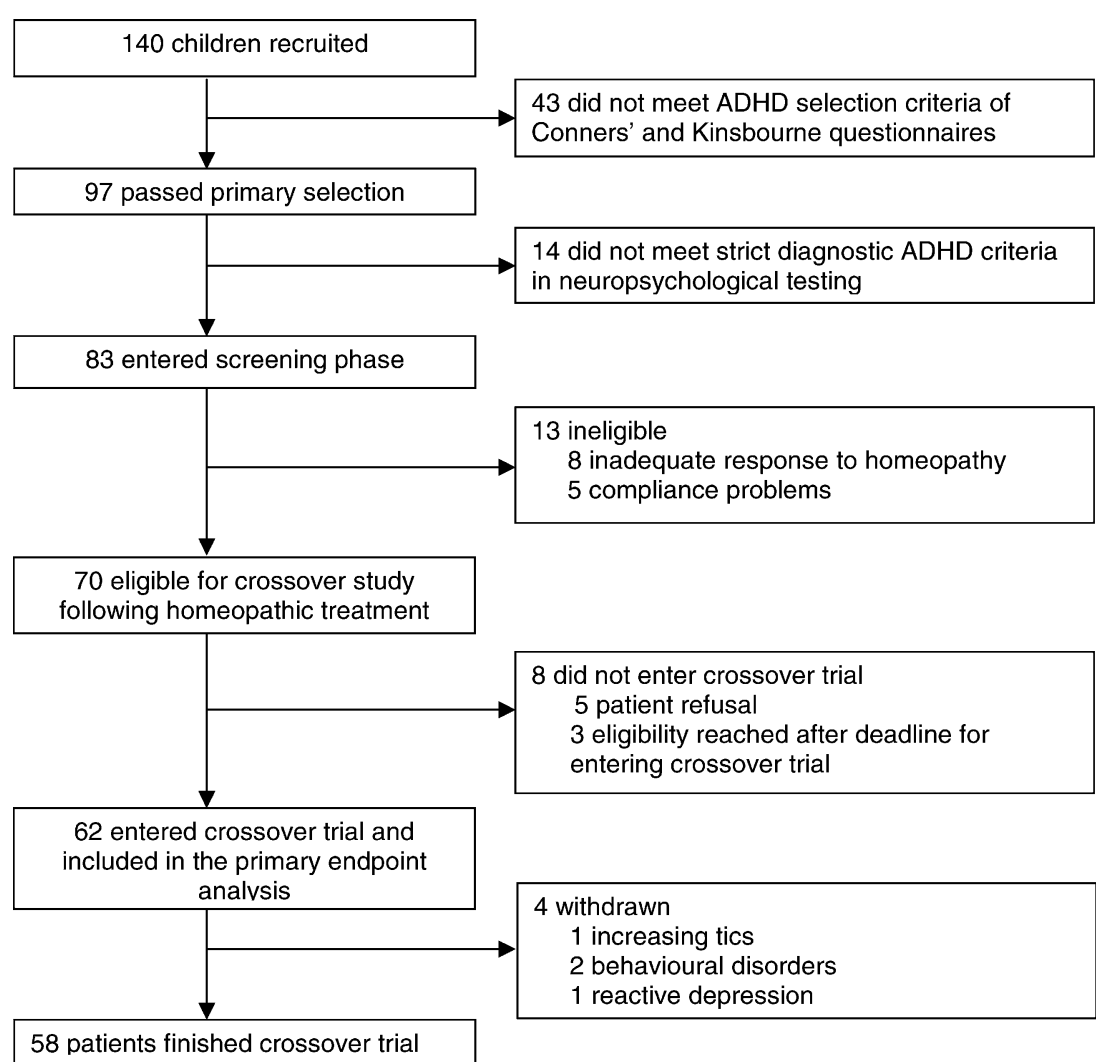


The assessments of each outcome variable recorded at the end of both crossover periods within a patient were not independent. To analyse such correlated data, a linear mixed model, including a random patient effect, a fixed treatment effect and a fixed period effect was applied using SAS version 8 [4,28]. With this approach, patients who dropped out after the first crossover period could also be included in the analysis by assuming missing at random. For other types of analyses, patients with missing values were excluded. For selected variables of behaviour and cognitive performance, the within-patient differences between diagnosis and the beginning of the crossover trial were analysed using the Wilcoxon signed rank test. The changes in CGI from diagnosis to later time points were analysed by paired $t$-test. All significance tests were two-sided. All analyses of secondary endpoints were of an exploratory nature without adjustment for multiple testing. The statistician was blinded to the sequence of the two study arms.

\section{Results}

Recruitment, participant flow and baseline data

A total of 140 children were evaluated and 83 fulfilled the rigorous criteria to enter the screening process. A group of 70 children was eligible for and 62 participated in the crossover study. Eight children dropped out due to insufficient response to homeopathy (three no response at all, five partial responders with a median improvement of 7 points, range 6-8) and five patients dropped out due to compliance problems (median improvement 6 points, range 3-8). All data presented below were collected from the 62 children who participated in the crossover trial. Figure 1 shows details of the recruitment phase and the participant flow. Summaries of the baseline data are presented in Table 1 and Table 2. Patient characteristics were similar between treatment arms (i.e. no statistically significant differences were found in baseline data between treatment arms with one exception, number recall (K-ABC, $P=0.0284)$. The median CGI before treatment was 19 (range 1525) on both treatment arms. As usual in groups of ADHD subjects, cognitive functions of the patients as a group did not deviate much from the normal population means (exception: auditory short term memory, Table 2).

\section{Screening phase}

The 62 children participating in the crossover trial attained the eligibility criteria after a mean treatment duration of 5.1 months (SD 3.20, range 1-18 months) with a median CGI of 8 (range $4-15$ ). The following homeopathic medications were used in potencies between Q 3 and Q 42 with success (number of patients): Calcarea-carb. (15), Sulphur (8), Chamomilla (5), Lycopodium (5), Silica (5), Hepar-sulph. (4), Nux-vom. (4), China (3), Ignatia (3), Mercurius (3), Capsicum (1), Causticum (1), Hyoscyamus (1), Phosphorus (1), Phosphoric-ac. (1), Sepia (1), Staphysagria (1) [1].

The neuropsychological tests assessed at diagnosis and at entry to the crossover trial allow comparison of performance throughout the screening phase. Highly significant improvement in visual detection of details, impulsivity and divided attention were observed (Table 3).

\section{Crossover trial}

Of the 62 randomised patients, 4 dropped out during the crossover trial, three during crossover period one and one during crossover period two (3 verum, 1 placebo). Reasons for drop out were increasing tics, behavioural problems and a reactive depression. Following the intention-to-treat principle, all 62 patients were included in the analysis.

Prior to the crossover trial, both treatment arms had similar CGIs (8, range 3-16 in arm A vs. 9, range 4-20 in

Table 1 Baseline data, diagnostic criteria and CGI data. No statistically significant differences were found in baseline data between treatment arms

\begin{tabular}{lll}
\hline & Arm A $(n=31)$ & Arm B $(n=31)$ \\
\hline Baseline data & & $3 / 28$ \\
Gender (girls/boys) & $4 / 27$ & $10(7-15)$ \\
Median age (range) & $10(7-15)$ & $20(65 \%) / 11(35 \%)$ \\
Social situation (intact family/single parent) & $23(74 \%) / 8(26 \%)$ & $4(13 \%)$ \\
Prior stimulant treatment & $7(23 \%)$ & \\
Clinical characteristics at diagnosis & & $29(94 \%)$ \\
DSM-IV criteria & $27(87 \%)$ & $17(55 \%)$ \\
Inattention $(n=56)$ & $19(61 \%)$ & $16(52 \%)$ \\
Hyperactivity/impulsivity $(n=36)$ & $15(48 \%)$ & $19(15-25)$ \\
Inattention and hyperactivity/impulsivity $(n=31)$ & $19(15-25)$ & $8(4-15)$ \\
CGI data & $8(5-14)$ & $58 \%$ \\
Median before treatment, cut-off $<14$ (range) & $58 \%$ & \\
Median at end of screening phase (range) & \\
Ratio of changes with respect to before treatment value & & \\
\hline
\end{tabular}


Table 2 Baseline data from neuropsychological tests ( $B C$ block counting, $D A$ divided attention, $G C$ gestalt closure, $G N$ go/nogo, $H M$ hand movements, $m$ mistakes, $M A$ matrix analogies, $N R$ number recall, $P S$ photo series, $S T M$ short-term memory, $T R$ triangles, $W O$ word order)

\begin{tabular}{|c|c|c|c|}
\hline & & $\operatorname{Arm~A}(n=31)^{\mathrm{a}}$ & $\operatorname{Arm~B}(n=31)^{\mathrm{a}}$ \\
\hline & Normal values & Baseline values (mean \pm SD) & \\
\hline \multicolumn{4}{|l|}{ Intelligence } \\
\hline General intelligence (K-ABC) & $100 \pm 15$ & $96.1 \pm 11.8$ & $95.2 \pm 7.7$ \\
\hline Sequential Processing Scale (K-ABC) & $100 \pm 15$ & $88.4 \pm 13.0$ & $85.5 \pm 10.0$ \\
\hline Simultaneous Processing Scale (K-ABC) & $100 \pm 15$ & $101.4 \pm 13.1$ & $101.8 \pm 10.4$ \\
\hline \multicolumn{4}{|l|}{ Memory } \\
\hline Sequential short-term memory (K-BC, HM) & $10 \pm 3$ & $8.5 \pm 3.2$ & $8.7 \pm 2.5$ \\
\hline Auditory abstract STM (K-ABC, NR) & $10 \pm 3$ & $7.6 \pm 2.0$ & $6.3 \pm 1.8$ \\
\hline Auditory semantic STM (K-ABC, WO) & $10 \pm 3$ & $8.3 \pm 2.9$ & $8.1 \pm 2.5$ \\
\hline Spatial short term memory (K-ABC, BC) & $10 \pm 3$ & $10.2 \pm 2.5$ & $9.7 \pm 2.9$ \\
\hline \multicolumn{4}{|l|}{ Perception } \\
\hline Visual global (K-ABC, GC) & $10 \pm 3$ & $10.0 \pm 2.6$ & $10.5 \pm 2.1$ \\
\hline Visual constructive (K-ABC, TR) & $10 \pm 3$ & $10.4 \pm 2.2$ & $9.9 \pm 2.5$ \\
\hline \multicolumn{4}{|l|}{ Executive functions } \\
\hline Inferential thinking (K-ABC, MA) & $10 \pm 3$ & $10.2 \pm 2.5$ & $10.5 \pm 2.2$ \\
\hline Performance sequences (K-ABC, PS) & $10 \pm 3$ & $10.1 \pm 2.4$ & $10.4 \pm 2.5$ \\
\hline \multicolumn{4}{|l|}{ Attention } \\
\hline Divided attention (TAP, DA, m) & $50 \pm 10$ & $48.4 \pm 11.7$ & $45.2 \pm 12.4$ \\
\hline Impulsivity (TAP, GN, m) & $50 \pm 10$ & $41.8 \pm 7.7$ & $41.9 \pm 11.6$ \\
\hline
\end{tabular}

${ }^{a}$ No statistically significant differences were found in neuropsychological baseline data between treatment arms (exception attributable to coincidental: number recall $(\mathrm{K}-\mathrm{ABC}) ; P=0.0284)$

arm B, Fig. 2, point 1). Within-patient comparison of treatment effect shows that the CGI, the primary endpoint, decreased under verum with a mean of 1.67 points (95\% CI -3.316, -0.016) compared with placebo, indicating a statistically significant improvement in ADHD symptoms with verum ( $P=0.0479$, Table 4$)$. The period effect was also statistically significant, with a positive parameter estimate of 2.19. The CGI was therefore on average 2.19 points higher in crossover period one, independent of treatment (12, range 3-24, verum in arm A compared with 13 , range 3-25, placebo in arm B, Fig. 2, point 2). At the end of crossover period two, ADHD symptoms decreased in children receiving verum (CGI 9, range 3-19) while children on placebo still had a high CGI (12, range 4-21) (Fig. 2, point 3). Also, at the end of the following 6 weeks of open label treatment, the ADHD symptoms of both treatment groups had returned to around their values at beginning of the crossover trial (8, range $2-16$ in arm A vs. 8 , range $2-21$ in arm B Fig. 2, point 4).
Exploratory analysis of 70 neuropsychological parameters during the crossover trial resulted in a few within-patient differences in behavioural and cognitive outcomes between placebo and verum, (Table 4): significantly better resistance to verbal interference in the VLMT during the verum phase $(P=0.0328)$ and a trend towards amelioration of stability of mood and in reaction to unexpected events were observed $(P=0.0693$ and $P=0.1001$, respectively). Conversely, verum resulted in a significant reduction in alertness to visual details (picture completion, WISC-III, $P=0.0302$ ) and a trend towards decreasing performance in visual-spatial organisation (bloc design, WISC-III, $P=0.0632$ ).

\section{Open label treatment follow-up}

Comparison of the scores CPRS scores between start of treatment and 14 weeks after crossover trial still revealed highly significant improvements in all subscales, in both

Table 3 Results of screening phase. Total of observed differences in cognitive performance between diagnosis and crossover period one (Wilcoxon signed rank test). ( $C$ median of reaction time, $G C A$ gestalt closure, $S D B$ standard deviation of reaction time)

\begin{tabular}{lllr}
\hline Outcome variable & Median of differences & Difference in \% ${ }^{\mathrm{a}}$ & $P^{\mathrm{b}}$ \\
\hline Visual global perception (GCA, K-ABC) & -3 & 10.1 & 0.0001 \\
Impulsivity (Go/NoGo, SDB, TAP) & -13 & 17.0 & Improvement \\
Impulsivity (Go/NoGo, median C, TAP) & 17.5 & 16.2 & 0.1044 \\
Impulsivity (Go/NoGo, mistakes, TAP) & -43 & 104.2 & 0.0001 \\
Divided attention (SDB, TAP) & -30.5 & 24.1 & 0.0001 \\
Divided attention (median C, TAP) & -32 & 44.3 & 0.0001 \\
Divided attention (missings, TAP) & -29 & 24.6 & 0.0001 \\
Divided attention (mistakes, TAP) & -30.5 & 27.1 & 0.0001 \\
Improvement \\
Improvement \\
Improvement \\
\hline
\end{tabular}

${ }^{a}$ Ratio of changes with respect to the before treatment values

${ }^{\mathrm{b}}$ If $P<0.05$, then the difference in performance between diagnosis and crossover period one is significantly different from zero 
Fig. 2 CGI course during crossover trial according to treatment arm (medians and ranges). Arm A: unexpected rise in CGI during verum phase, possibly due to expectation of receiving placebo in first phase. Persistent high CGI with placebo in second phase, normalisation of CGI with open label verum after crossover trial. Arm B: CGI rose with placebo in first phase and returned to CGI values within the normal range with verum treatment in second phase, as expected

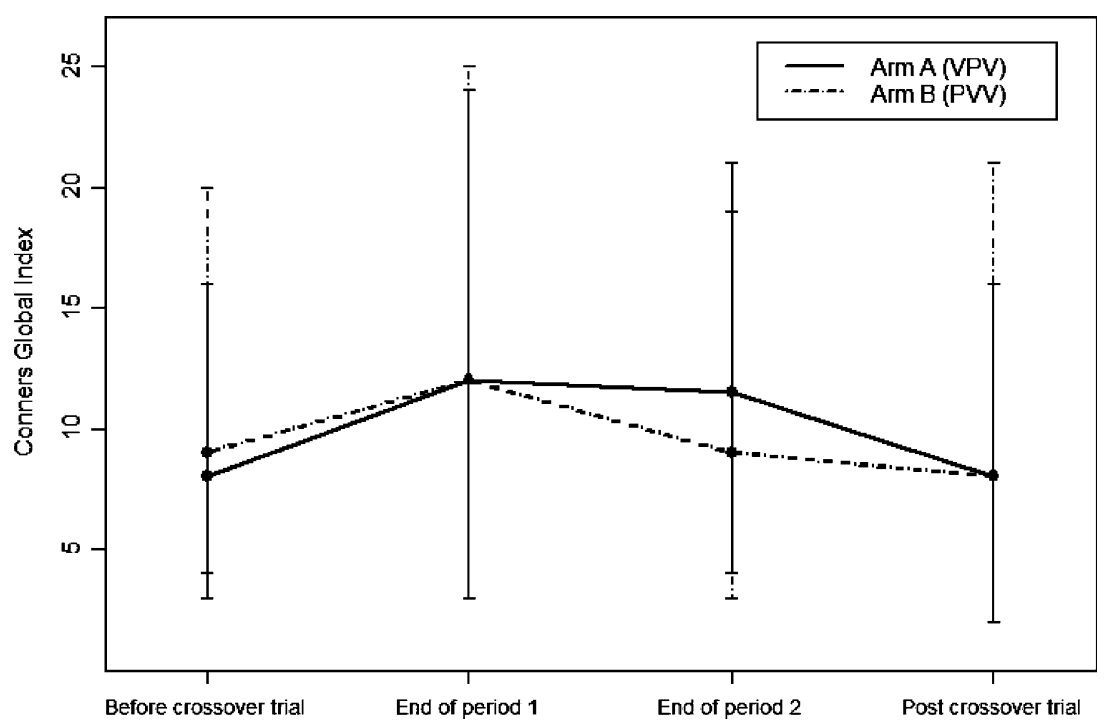

mothers' and fathers' ratings. Teachers ratings (CTRS) showed a significant improvement in behaviour, and a trend in improvement of the CGI. Impulsivity/hyperactivity and passivity were improved, but did not reach significance in the teachers observations (Table 5). The median CGI of the 62 children dropped significantly from 19 (range 15-25) at treatment start to 8 (range 216) 6 weeks after the crossover trial (Fig. 3). At followup 14 weeks after the crossover trial, however, a rise to 10 points (range $1-18$ ) was observed (Fig. 3). This rise may be attributable to lack of treatment compliance as both children and parents were less likely to follow the strict conditions of the crossover periods during continued open label treatment. At the final assessment (median duration of treatment 19 months, range 10-30 months: early entry into the trial led to longer follow-up times), 53 children reached a median CGI of 7 points (range 2-15) (Fig. 3), corresponding to an overall improvement of $63 \%(P<0.0001)$.

\section{Discussion}

The necessity of individual prescriptions in homeopathy is a severe obstacle for any double blind trial. The present study design, including a screening phase prior the crossover trial, and analysing deterioration instead of improvement during crossover trial, enabled us to use individually prescribed medication in a double blind trial. Since it is known that homeopathic medications may produce strong carryover effects and that these effects may compromise the results of a crossover trial, placebo-phases had to be of a duration which was at the limits of tolerance for a number of participating families. Another limitation of the study design was the necessity to exclude patients from the crossover trial who showed an inadequate response to homeopathic treatment during the screening phase. This means that the intention to treat principle could not be followed through all the way. The implications of this obstacle are discussed

Table 4 Results of crossover trial. Parameter estimates and $P$ values of linear mixed models for outcome variables. ( $B D$ bloc design, $P C$ picture completion)

\begin{tabular}{lllll}
\hline Outcome variable & Effect $^{\mathrm{a}}$ & Parameter estimate & $P$ & Effect during verum period \\
\hline CGI A (primary endpoint) & Treatment & -1.67 & 0.0479 & Improvement \\
Verbal loss after interference B (VLMT) & Period & 2.19 & 0.0102 & Improvement \\
Stability of mood C (QCB) & Treatment & -11.27 & 0.0328 & 0.6294 \\
& Period & -2.52 & 0.0693 & Improvement \\
Reaction to unexpected events D (QCB) & Treatment & 0.45 & $<0.0001$ & Improvement \\
Alertness to visual details E (PC, WISC-III) & Period & -1.07 & 0.1001 & 0.0003 \\
Visual spatial organisation F (BD, WISC-III) & Treatment & 0.29 & 0.0302 & Worsening \\
& Treatment & -0.67 & 0.0013 & Worsening \\
\hline
\end{tabular}

\footnotetext{
${ }^{a}$ Treatment effect refers to within-patient mean difference between treatments, i.e. verum-placebo. Period effect refers to within-patient mean difference between periods, i.e. period 1-period 2. Tests A and B measure intensity of symptoms, negative treatment effect implies improvement. Tests C, D, E and F measure capabilities, positive treatment effect implies improvement
} 
Table 5 Long-term treatment effects. Differences in CPRS and CTRS between diagnosis and follow-up 14 weeks after crossover trial (Wilcoxon signed rank test)

\begin{tabular}{|c|c|c|c|c|}
\hline CPRS & Median of differences & Difference in $\%$ & $P$ & Effect \\
\hline Behaviour & 3.5 & 42.5 & 0.0001 & Improvement \\
\hline Learning/attention & 3 & 36.5 & 0.0001 & Improvement \\
\hline Psychosomatics & 1 & 46.7 & 0.0004 & Improvement \\
\hline Impulsivity/hyperactivity & 3 & 41.5 & 0.0001 & Improvement \\
\hline Shyness/anxiety & 1 & 39.5 & 0.0001 & Improvement \\
\hline CGI & 7 & 43.3 & 0.0001 & Improvement \\
\hline \multicolumn{5}{|l|}{ CTRS } \\
\hline Behaviour & 3 & 36.4 & 0.0347 & Improvement \\
\hline Impulsivity/hyperactivity & 2 & 28.0 & 0.1932 & Improvement \\
\hline Passivity & 2 & 27.8 & 0.1184 & Improvement \\
\hline CGI & 3 & 30.8 & 0.0561 & Improvement \\
\hline
\end{tabular}

below. Patients participating in the study were selected by a rigorous diagnostic evaluation, ruling out any other diagnosis but ADHD. In addition, strict inquiries at periodical monitoring were performed to prevent the application of any other treatment but homeopathy. The long duration of observation of an average of 19 months was chosen to grant that observed changes are not just of transitory nature.

Effects of homeopathy in children with ADHD have been demonstrated in the present randomised double blind crossover trial. The within-patient difference in CGI between treatment with verum or placebo was statistically significant $(P=0.0479)$ and supports the positive effect of homeopathy. However, CGI decreased by only $17 \%$ (1.67 CGI points), somewhat less than that expected from the results of an earlier study [8]. This may be partially due to more precisely prescribed individual homeopathic medication in this trial, with a consequently stronger carry-over effect. Another reason for this small difference is the unexpected increase in CGI during crossover period one in both treatment groups. This increase could possibly be explained by a psychological expectancy effect [26]. Parents and children both may have anticipated placebo in crossover period one rather than two, and this presumed expectancy (period) effect was stronger than the treatment
Fig. 3 Course of CGI between diagnosis and follow-up (median, quartile, range). Alterations of CGI throughout screening-phase, crossover trial and long-term follow-up.

Transient rise after crossover trial due to possible compliance problems. The latest follow-up during open label treatment showed a maximum amelioration of 12 points $(63 \%)$

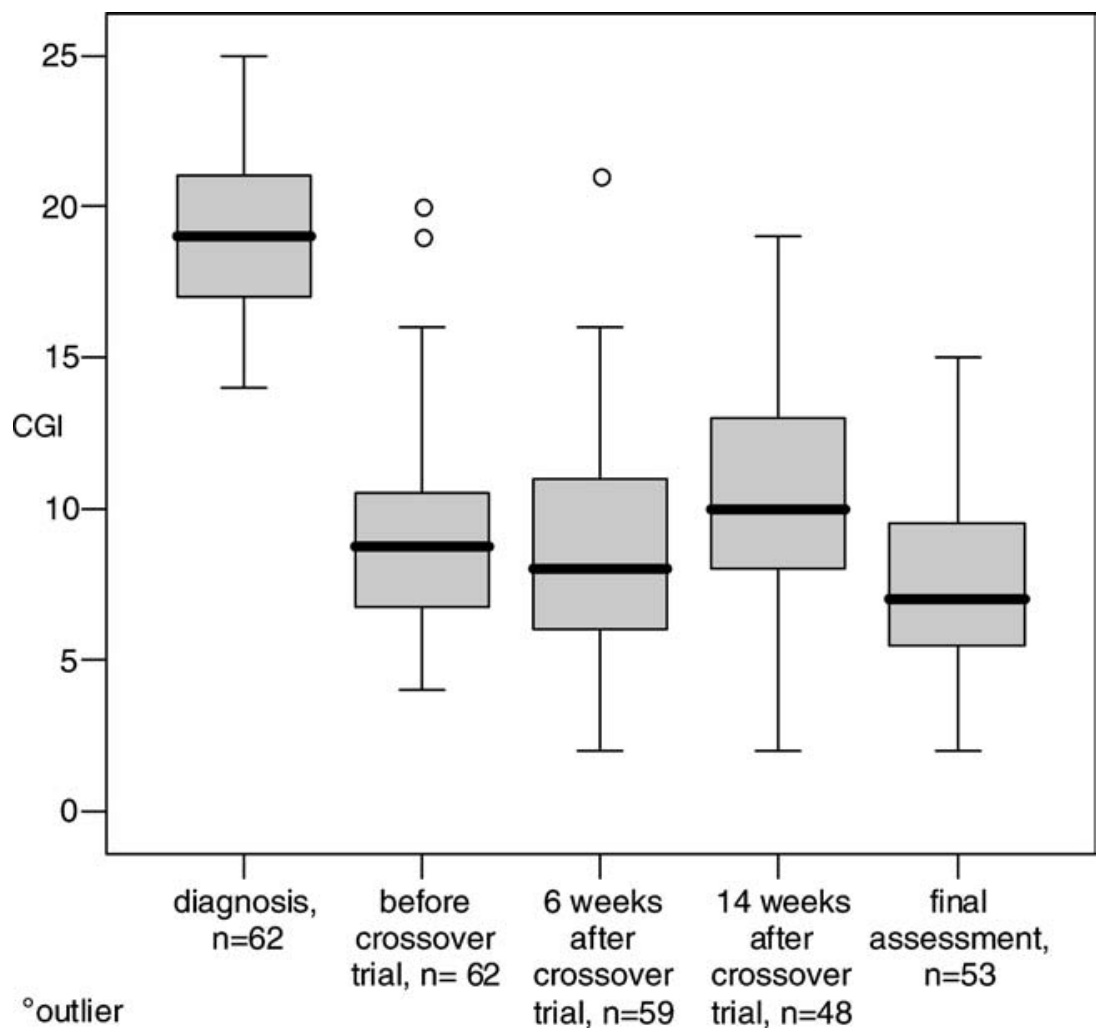


effect. The question arises whether the parents just rated the CGI in a way which they expected to be favourable for the outcome of the study. While on treatment arm A during the first crossover period this might have actually been the case, such a bias can be ruled out for crossover period two on arm A, in which the same parents reported a persistent deterioration under placebo. After the initial (unexpected) worsening under verum, they would report an improvement in crossover period two, if their rating would follow just their opinion about what should be a favourable outcome. However, in crossover period two, the phenomenon disappeared and led to a significant decrease in the CGI of the verum group (treatment effect) whereas the placebo group maintained an elevated CGI. The intended treatment effect and the unexpected period effect together resulted in the observed CGI levels, especially in crossover period one. The treatment effect became evident in crossover period two. Following an additional 6 weeks of open label treatment, both groups had CGI values similar to those at the beginning of the crossover trial, an indirect confirmation of the appropriateness of the study design. The validity of the CGI has been proven over time across many studies and therefore won't be doubted in the context of this study [25].

Since at the beginning of the trial it was unknown whether homeopathic treatment would affect cognitive functioning and which functions might possibly be affected, a large number of cognitive variables, often impaired in ADHD children, were selected for study. The trial design inevitably required a repetition of these tests within a short period of time. Consequently, learning effects, which might mask possible treatment effects, were to be expected. Only a few significant treatment effects were observed (positive: improved auditory shortterm memory, a trend to increased stability of mood, better reactions to unexpected events; negative: decrease in alertness to visual details and the trend towards decreased visual spatial organisation). These significant changes in neuropsychological tests during the crossover trial may be due to chance only, as a result of the high number of tests performed. The expected learning effects are indicated by significant period effects of many variables. A strong carry-over effect with a persistence of $66 \%$ of the improvements reached under homeopathy throughout the placebo phases, however, diminished the possibility of positive neuropsychological tests further. Eleven outliers in crossover period one and six in crossover period two showed CGI-values contrary to the expected courses, and diminished the statistical results additionally in a way that the CGI difference between placebo and verum was considerably smaller than expected.

Comparison of some of the cognitive tests at diagnosis and at the beginning of the crossover trial after the initial open label homeopathic therapy suggests, however, highly significant treatment effects. Since every other treatment was stopped during the screening phase, this improvement is only explicable by the homeopathic intervention. The question arises whether the eight patients who dropped out of the screening phase due to insufficient treatment response cause a selection bias influencing the results considerably. Five of these dropouts still had a partial amelioration under treatment. Their influence on the high significance of the results of the screening phase and the long-term follow up would therefore have been minimal. Thus, it is unlikely that they would have caused considerably different results. Improvements in cognitive deficits following stimulant treatment of ADHD patients, especially spatial working memory, attention-set shifting and visual search performance have been reported [21]. The effects of homeopathy observed in this study are similar. To gain a better insight into the quantitative aspects of cognitive improvements following medication, a comparison of matched patients receiving stimulants, homeopathy or placebo would be worthwhile.

The CGI and the CPRS scores (parent ratings) decreased between $37 \%$ and $63 \%$ over the long-term observation period, most probably related to treatment induced adjustment of behaviour. The CTRS improvement ratings by teachers were smaller (between $28 \%$ and $37 \%$ ) than those of the parents, reflecting the higher cognitive stress for patients in school situations. However, on the whole, the overall intensity of ADHD symptoms appears to be lower during treatment and results in an improvement in the childrens' social, emotional and scholastic behaviour. The question whether these long-term improvements are a treatment effect or merely due to a spontaneous change in development of the children cannot by definitely answered by our trial data. However, the authors have observed that stopping treatment after prolonged medication leads to a slow decline of previous improvements over a time period of several months in most children. Such a course cannot just be explained by spontaneous evolution and is highly suggestive of a treatment effect.

The results of this trial point to the effectiveness of homeopathy in the treatment of ADHD. To corroborate the findings presented here, the authors suggest a larger and independent multicentre study. Since homeopathic treatment of ADHD is not an easy undertaking, a training phase for participating treating physicians in the methodology used in this study would be essential prior to starting the trial. Different homeopathic approaches might lead to other observations. Factors limiting the widespread use of homeopathy in the treatment of ADHD are the time needed for amelioration of symptoms, thus excluding this treatment from use in emergency situations, and the necessity of accurate observation and recording of symptoms by the parents.

Acknowledgements We sincerely thank all patients and families who took part in this trial for the enormous amount of time they sacrificed for travelling, testing and treatment sessions as well as for their patience in observing symptoms. Sincere thanks also to Jacqueline Ryffel and her staff at Spagyros for preparing and mailing the medications and placebos to the patients. Many thanks to Martina Frei for her contribution in the starting phase of the study 
and to Carol Lim for her substantial help in the preparation of the manuscript. This study was financed by grants of the following foundations and institutions: Gertrude von Meissner Foundation, Basel; Software AG Foundation, Darmstadt; Hans Eggenberger Foundation, Zürich; SNE Foundation, Solothurn; Fondation Homéopathie Pierre Schmidt Genève; PanMedion Foundation, Zürich; Spagyros AG, Gümligen; Gudjons Laboratory, Stadtbergen; Swiss Federal Railways (SBB), Berne.

\section{References}

1. Allen TF (1990) The Encyclopaedia of Pure Materia Medica, vol.. Jain, New Delhi

2. American Psychiatric Association (1994). Diagnostic and statistical manual of mental disorders, DSM-IV, 4th edn. American Psychiatric Association, Washington,

3. von Bönninghausen C (2000) Bönninghausens therapeutisches Taschenbuch, revidierte Ausgabe. Hrsg. K-H. Gypser, Stuttgart

4. Brown H, Prescott R (1999) Applied mixed models in medicine. Wiley, Chichester, pp 261-294

5. Brue AW, Oakland TD (2002) Alternative treatments for attention deficit/hyperactivity disorder: does evidence support their use? Altern Ther Health Med 8: 68-74

6. Carte ET, Nigg JT, Hinshaw SP (1996) Neuropsychological functioning, motor speed, and language processing in boys with and without ADHD. J Abnorm Child Psychol 24: 481-498

7. Conners CK(1997) Conners rating scales (revised), technical manual. MHS, Toronto

8. Frei H, Thurneysen A. Treatment for hyperactive children: homeopathy and methylphenidate compared in a family setting. Brit Hom J 2001; 90: 183-188.

9. Grizzle JE (1965) The two-period change-over design and its use in clinical trials. Biometrics 21: 467-480

10. Gross-Tsur V, Lahad A, Shalev R (2003) Use of complementary medicine in children with attention deficit hyperactivity disorder and epilepsy. Pediatr Neurol 29: 53-55

11. Hahnemann CFS (1989) Organon der Heilkunst, 6th edn. Haug, Heidelberg

12. Helmstaedter C, Lendt M, Lux S (2001) Verbaler Lern- und Merkfähigkeitstest (VLMT). Beltz Test Gmbh, Göttingen

13. Kaufman AS, Kaufman NL (1983) Kaufman Assessment Battery for Children (K-ABC). AGS Publishing, Circle Pines

14. Kinsbourne M (1973) School problems. Paediatrics 52: 679-710

15. Kleijnen J, Knipschild P (1991) Clinical trials of homeopathy. BMedJ 302: 316-323
16. Lahey BB, Applegate B, McBurnett K, Biederman J, Greenhill L, Hynd GW, Barkley RA, Newcorn J, Jensen P, Richters J (1994) DSM-IV field trials for attention deficit hyperactivity disorder in children and adolescents. Am J Psychiatry 151: $1673-1685$

17. Lamont J (1997) Homeopathic treatment of attention deficit disorder. Br Hom J 86: 196-200

18. Largo R (1993) Zürcher neuromotorische Untersuchung. Abteilung für Wachstum und Entwicklung, Universitäts-Kinderklinik, Zürich

19. Lattmann P, Schneider A (1995) Informationen für den Prüfer zur Durchführung klinischer Versuche mit Heilmitteln in der Schweiz. SGCI, Zürich

20. Linde K, Clausius N, Ramirez G, Melchart D, Eitel F, Hedges LV, Jonas WB (1997) Are the clinical effects of homeopathy placebo effects? A meta-analysis of controlled trials. Lancet 350: 834-843

21. Mehta MA, Goodyer IM, Sahakian BJ (2004) Methylphenidate improves working memory and set shifting in $\mathrm{AD} / \mathrm{HD}$ : relationships to baseline memory capacity. J Child Psychol Psychiatry 45: 293-305

22. Minder P (2003) The Q-potencies, a comprehensive and profound way of medication in homeopathy. Schweiz Zschr GanzheitsMedizin 15: 348-353

23. Seidman LJ, Biederman J, Monuteaux MC, Weber W, Faraone SV (2000) Neuropsychological functioning in nonreferred siblings of children with attention deficit/hyperactivity disorder. J Abnorm Psychol 109: 252-265

24. Senn S (1993) Cross-over Trials in Clinical Research. Wiley, Chichester, pp 12-13

25. Snyder SM, John F, Xenakis SN (2004) Validation of ADHD rating scales. J Am Acad Child Adolesc Psychiatry 43: 11891190

26. Stewart-Williams S, Podd J (2004) The placebo effect: dissolving the expectancy versus conditioning debate. Psychol Bull 130: $324-340$

27. Tewes U, Schallberger U, Rossmann K (1999) Hamburg Wechsler Intelligenztest für Kinder III (HAWIK-III). Hogrefe, Göttingen

28. Verbeke G, Molenberghs G (1997) Linear mixed models in practice. Springer, Berlin Heidelberg New York

29. World Medical Association (2002) Declaration of Helsinki. Washington

30. Zimmermann P, Fimm B (1992) Testbatterie zur Aufmerksamkeitsprüfung TAP, Handbuch. Psychologische Testsysteme PsyTest, Freiburg 